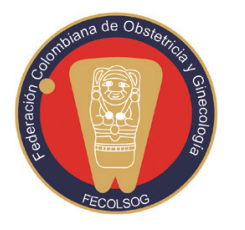

\title{
COLOMBIA Y EL CUMPLIMIENTO DEL OBJETIVO DE DESARROLLO DEL MILENIO RESPECTO A LA SALUD MATERNA
}

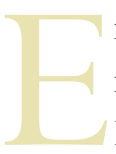

n el año 2000, Colombia refrendó la Declaración del Milenio de la Organización de las Naciones Unidas, en donde 189 Estados se comprometieron en el cumplimiento de los "Objetivos de Desarrollo del Milenio" (ODM). Estos ODM están soportados en los principios de equidad, igualdad y humanización como bases para el desarrollo humano como fin y medio de las políticas del Estado. Con respecto al cumplimiento del objetivo 5: "Mejorar la salud materna", que fijó la meta universal de reducir la mortalidad materna en tres cuartas partes en el periodo 1990-2015, Colombia definió como propósito reducirla a 45 defunciones maternas por cien mil nacidos vivos usando como base la tasa registrada en el año 1998 (1).

Para el año 2014, la razón de mortalidad materna reportada por el Departamento Administrativo Nacional de Estadística (DANE), de manera preliminar, fue de 53,4 por cien mil nacidos vivos, correspondientes a 344 muertes maternas tempranas, lo que equivale a un $85 \%$ del cumplimiento de la meta (2) (tabla 1).

En el periodo 2011-2014, la reducción de muertes maternas tempranas fue importante, a diferencia de

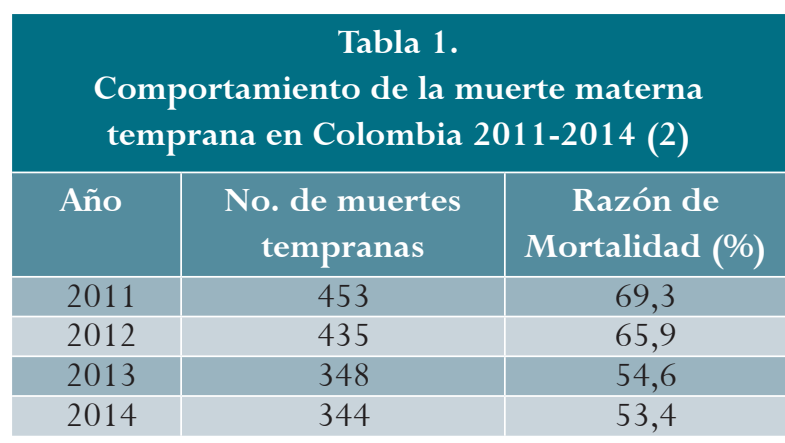

lo observado entre los años 2006-2010, donde la mortalidad materna estuvo prácticamente estable, pasando de 75 a 71 por cien mil nacidos vivos al final de ese quinquenio (2).

El comportamiento observado en la semana epidemiológica 24 del 2015, reportado en el Boletín epidemiológico del Instituto Nacional de Salud (INS), muestra un promedio de 6,0 muertes tempranas por semana, por debajo del promedio calculado para cumplir el objetivo de desarrollo del milenio, que es de 6,5, lo cual nos permite inferir que, de mantenerse esta tendencia, es factible cumplir la meta trazada para Colombia de 45 defunciones maternas por cien mil nacidos vivos (3).

Este comportamiento es el resultado de intervenciones como:

- Acceso a los métodos anticonceptivos modernos al $75 \%$ de mujeres en edad fértil (4) (sexto país de América en lograr esa prevalencia de uso).

- Cobertura del 99\% de atención del parto institucional, en el $99 \%$ de los casos por personal calificado (5).

- Desarrollo de normas técnicas y guías de atención para la detección temprana de las alteraciones del embarazo, y atención del parto normal y distócico, adoptando los principios del parto humanizado, la atención de la embarazada menor de 15 años, la atención preconcepcional y la interrupción voluntaria del embarazo (6).

Las Metas de Desarrollo Sostenible fijan ahora como meta para el año 2030, en países como Colom- 
bia, la reducción en un $75 \%$ de la mortalidad materna registrada en el año 2010, es decir, se deberá alcanzar una razón de mortalidad materna de 24 por cien mil nacidos vivos (7). No obstante, permanecen vigentes una serie de determinantes que inciden negativamente en la salud materna $(8,9)$ los cuales, de no abordarse adecuadamente, desacelerarán la tendencia observada en el último quinquenio y harán poco factible el cumplimiento de este nuevo objetivo.

Se hace entonces prioritario incidir en los determinantes estructurales (10), apuntando a intervenir, en primer lugar, la desigualdad que se expresa en un mayor riesgo de muerte materna en las edades extremas de la vida reproductiva, siendo más alta en menores de 14 años (52,4 por cien mil nacidos vivos) y en mayores de 35 años (140,6 por cien mil nacidos vivos); en mujeres residentes en el área rural (riesgo relativo de 2,1) debido a barreras geográficas o de orden público que limitan el acceso, deficiencias en la infraestructura vial y en las comunicaciones en general (5); en mujeres del régimen subsidiado (66\% de los casos) y en mujeres sin escolaridad o escolaridad primaria (una de cada tres muertes maternas) (10); estos factores condicionan grandes brechas regionales, las cuales dieron como resultado departamentos que en el año 2014 reportaron razones de mortalidad materna por encima de 140 por cien mil nacidos vivos, como Chocó, Putumayo y La Guajira, y razones inaceptables superiores a 300 por cien mil nacidos vivos en Guaviare, Guainía y Vichada (2).

Estas desigualdades tienen relación directa con la debilidad en la implementación y apropiación de las políticas en salud materna, razón por la que los entes territoriales deberán garantizar la integralidad de las acciones a lo largo del proceso preconcepcional, la gestación y el posparto, para lo cual estos deberán comprometerse con la implementación de todas las intervenciones que Colombia ha desarrollado en el contexto de la maternidad segura.

Colombia ha desarrollado protocolos de atención preconcepcional (11) en respuesta al cambio del perfil epidemiológico de la muertes maternas, donde se evidencia un claro incremento de las causas indirectas asociadas a problemas oncológicos, cardiopatías, complicaciones neurológicas y enfermedades autoinmunes, entre otras $(5,12)$.

De igual manera, en este contexto de políticas públicas se han desarrollado, con una amplia participación de la academia y las sociedades científicas, normas técnicas y guías de atención para la detección temprana de las alteraciones del embarazo, la atención del parto normal y distócico, adoptando los principios del parto humanizado (13), la atención de la embarazada menor de 15 años (14) y las guías para la interrupción voluntaria del embarazo en las situaciones previstas en la sentencia C-355/06 (15).

Dado que el $60 \%$ de las causas de muerte materna están asociadas a la triada trastornos hipertensivos, complicaciones hemorrágicas y sepsis (16), y que en el $90 \%$ de los casos se presentan fallas durante el proceso de la atención relacionadas con el manejo inadecuado de estas situaciones de emergencia obstétrica (17), desde el año 2010, en una alianza entre el Ministerio de Salud y la Federación Colombiana de Obstetricia y Ginecología (FECOLSOG), se elaboró y está en proceso de implementación un modelo de seguridad clínica para la atención de las emergencias obstétricas (18).

Este modelo incorpora la evaluación de la capacidad resolutiva de las instituciones que atienden estas emergencias, y la detección y el análisis de casos de morbilidad materna extrema, como elementos sustanciales para la identificación de oportunidades de mejora que nos permitan avanzar en la cobertura a la calidad de la atención, e incorporar herramientas para facilitar la ejecución de los planes de mejora, como listas de chequeo, diagramas de flujo para el manejo de las emergencias, kits con los insumos necesarios y actividades de capacitación para el reconocimiento, manejo y adecuada referencia de estas morbilidades en una iniciativa denominada "Prácticas claves que salvan vidas".

Podríamos colegir que la política pública contiene los elementos primordiales para seguir avanzando; 
lamentablemente, su no implementación y apropiación van ligadas a la pobreza, a la poca transparencia en el uso de los recursos en las regiones más críticas del país (14 veces mayor mortalidad materna) (8), al desinterés y poco compromiso de algunos aseguradores, y a la deficiente remuneración para prestadores y profesionales de la salud involucrados en la atención materna.

Es paradójico que los avances de nuestro país en materia de políticas públicas sean un ejemplo que se debe seguir por los demás países de América Latina, y que la continuidad de este proceso no esté garantizada, más aún cuando el quehacer está claro (19).

Se requiere, entonces, que para el Gobierno nacional este sea un tema esencial, siendo preocupante el que a pesar de estar incluida la reducción de la mortalidad materna como una meta del plan de desarrollo 2015-2017, la inclusión de las acciones de salud materna dentro de un enfoque materno-infantil, como si fuera un asunto accesorio a este y no uno derivado del goce efectivo de los derechos sexuales y reproductivos, desdibuje los avances para focalizar la garantía, promoción y protección de los derechos de las mujeres como la piedra angular de la intervención para reducir la morbilidad materna grave y la mortalidad materna (20).

\section{Edgar Iván Ortiz-Lizcano, MD, MSc}

Especialista en Obstetricia y Ginecología

Máster en Salud Pública

Fellow del Colegio Americano de Ginecología

y Obstetricia

Presidente de la Federación Colombiana de

Obstetricia y Ginecología (FECOLSOG)

Presidente de la Federación Latinoamericana

de Sociedades de Obstetricia y Ginecología

(FLASOG)

\section{REFERENCIAS}

1. Documento Conpes Social 140, Consejo Nacional de Política Económica y Social. Colombia: Departamento Nacional de Planeación; 2011.
2. Departamento Administrativo Nacional de Estadística (DANE). Estadísticas vitales (defunciones y nacimientos). Muertes maternas notificadas a Sivigila (semana epidemiológica 24 del 2015). Bogotá: DANE; 2015.

3. Instituto Nacional de Salud (INS). Informe del evento Mortalidad Materna a semana 24. Bogotá: INS; 2015.

4. Profamilia - Ministerio de Salud y Proteccion Social. Encuesta Nacional de Demografia y Salud. Bogotá: Profamilia - Ministerio de Salud y Proteccion Social; 2010.

5. Departamento Nacional de Planeación (DNP). Informe de seguimiento de los Objetivos de Desarrollo del Milenio. Bogotá: DNP; 2014.

6. Prada E, Singh S, Remez L, Villareal C. Embarazo no deseado y aborto inducido en Colombia. Causas y consecuencias. Nueva York: Guttmacher Institute; 2011.

7. United Nations, General Assembly. Report of the Open Working Group of the General Assembly on Sustainable Development Goals. General Assembly, 68 session. New York: United Nations; 2014.

8. Houweling TA, Ronsmans C, Campbell OM, Kunst AE. Huge poor-rich inequalities in maternal care, an international comparative study of maternity and child care in developing countries. Bull World Health Organ. 2007;65:745-54.

9. Cárdenas LM, Cotes K, Chaparro PE, Fernández JA, Paternina A, Castañeda C, et al. Maternal Mortality in Colombia in 2011: a two level ecological study. PLoS One. 2015;10:e0118944.

10 Ministerio de Salud y Protección Social. ASIS - Análisis de la situación de salud, Colombia 2013. Bogotá: Ministerio de Saud y Proteccion Social; 2013.

11. Ministerio de Salud y Protección Social, Dirección de Promoción y Prevención. Protocolo de atención preconcepcional. Bogotá: Ministerio de Salud y Protección Social; 2014.

12. Lumbiganon $P$, Laopaiboon $M$, Intarut $N$, Vogel JP, Souza JP, Gülmezoglu AM, et al. Indirect causes of severe adverse maternal outcomes: a secondary analysis of the WHO Multicountry Survey on Maternal and Newborn Health. BJOG. 2014;Suppl 1:32-9.

13. Colciencias - Ministerio de Salud y Protección Social. Guía de Práctica Clínica (GPC) para la prevención, detección temprana y tratamiento de las alteraciones del embarazo. Bogotá: Colciencias - Ministerio de Salud y Proteccion Social; 2013. 
14. Ministerio de Salud y Protección Social - Fondo de Población de las Naciones Unidas. Protocolo de atención a la embarazada menor de 15 años. Bogotá: Ministerio de Salud y Protección Social - Fondo de Población de las Naciones Unidas; 2014.

15. Ministerio de Salud y Protección Social - Fondo de Población de las Naciones Unidas. Prevención del aborto inseguro en Colombia. Protocolo para el sector salud. Bogotá: Ministerio de Salud y Protección Social - Fondo de Población de las Naciones Unidas; 2014.

16. Say L, Chou D, Gemmill A, Tunçalp Ö, Moller AB, Daniels J, et al. Global causes of maternal death: a WHO systematic analysis. Lancet Glob Health. 2014;2: e323-33.

17. Fundación Santafé de Bogotá CEIS - Ministerio de Salud y Protección Social - Fondo de Población de las Naciones Unidas. Análisis descriptivo de los casos de mortalidad materna 2007, de evaluación de impacto de las estrategias desarrolladas en el nivel nacional y regional para la reducción de la mortalidad materna en el país en el marco del SGSSS. Bogotá: Fundación
Santafé de Bogotá CEIS - Ministerio de Salud y Protección Social - Fondo de Población de las Naciones Unidas; 2007. p. 131-36.

18. Guerrero J, Ortiz E, Sarria O, Gallego G. Modelos de seguridad para la atención de la emergencia obstétrica en instituciones de salud. Bogotá: Ministerio de Salud y Protección Social - Fondo de Población de las Naciones Unidas; 2014.

19. Colombia es ejemplo de salud materno-infantil en México. Junio 2012. [En línea]. [Visitado 2014 Jun 25]. Disponible en: http://www.elhospital.com/eh/ secciones/EH/ES/MAIN/N/NOTICIAS2/doc_88202 HTML.html?idDocumento $=88202$.

20. López A, Benia W, Contera M, Guida C, Echeveste L. Del enfoque materno infantil al enfoque de la salud reproductiva, de salud, género, derechos sexuales y reproductivos. Diálogos entre la universidad y organizaciones de la sociedad civil. Montevideo: Cátedra Libre en Salud Reproductiva, Sexualidad y Género, Universidad de la República; 2003. p. 175-218. 